\title{
Español para la tercera edad: resignificación y valoración social de alumnos y profesores
}

\author{
Myrian Vasques Oyarzabal \\ myrian.ead@gmail.com \\ Vera Regina Aquino Vieira \\ veraav29@gmail.com \\ Adelia Broering Koerich \\ adeliabk@gmail.com \\ Mirella Nunes Giracca \\ mirellagiracca@gmail.com
}

\section{Resumen}

La educación a personas de la tercera edad cobra cada vez más importancia, sobretodo en tiempos de descubiertas y tecnologías que alargan la vida humana. En este sentido, y para dar más sentido a la existencia, la enseñanza debe ser pensada como un derecho para todas las personas, de todas las edades, y debe abarcar todos los ámbitos y aspectos de la existencia. El presente trabajo tiene como objetivo presentar el proyecto La Enseñanza de Español para la Tercera Edad, desarrollado desde 2004 en parcería entre el Núcleo de Estudos para a Terceira Idade y el Núcleo de Suporte Pedagógico para Professores de Línguas Estrangeiras de la Universidade Federal de Santa Catarina (NETI-NUSPPLE/UFSC). Presentamos aquí la experiencia desarrollada durante ocho años, período que comprende desde el inicio hasta el momento actual, como también la metodología, el abordaje y las estrategias y materiales adoptados para llevar a cabo la investigación, extensión y docencia para un público específico. Destacamos la importancia de la educación a lo largo de la vida como una de las posibilidades de inclusión y de recuperación del locus social de las generaciones mayores en la sociedad, pues tenemos como principio que los adultos mayores tienen la misma capacidad de aprender que en otras épocas, y que las variaciones obedecen a conocimientos previos, tiempos de cada uno, interés, motivaciones y en gran medida de las condiciones en que ocurre.

Palabras-clave: Enseñanza aprendizaje. Tercera edad. Géneros textuales. Mapas semánticos.

\section{Spanish for the elderly: a new social enhancing and meaning for students and teachers}

\begin{abstract}
Educating elder people is increasingly requiring more importance especially in this period of dis coveries and technologies which extend the human life time. In this sense, for making more sense to the
\end{abstract}


human existence, the education must be thought as a right for all people of every age and it has to involve all scopes and aspects of existence. This paper aims to present the Project La Enseñanza de Español para la Tercera Edad (Teaching Spanish for the Elderly), which has been developed since 2004 in partnership with Núcleo de Estudos para a Terceira Idade (Centre of Studies of Elder People) and Núcleo de Suporte Pedagógico para Professores de Línguas Estrangeiras (Centre of Teaching Support for Teachers of Foreign Languages) of Universidade Federal de Santa Catarina (NETI-NUSPPLE/UFSC). We here present an experiment developed for eight years; this period reaches the very beginning of the project and goes to the current days. We also expose the methodology, approach, strategies and materials used to carry on our research, extension and teaching for a specific public. We highlight the importance of education throughout the life as one of the possibilities of including and recovering the social location of elder generations in our society because we understand that older people have as much ability of learning as in other times of their lives and variations respond to previous knowledge, each person's time of learning and it depends a lot on the conditions it happens.

\section{Key-words: Teaching and Learning. Elderly. Textual Genres. Semantic Maps.}

\section{Introducción}

El aumento de la población mayor de sesenta años en Brasil hace parte de un fenómeno mundial sin precedentes en la historia de la humanidad. El boletín no 12/ octubre - 211 del Observatorio Demográfico (CELACD), apunta el envejecimiento de la población "como un cambio profundo que tiene repercusiones en todas las facetas de la vida humana", y destaca Brasil por el acelerado crecimiento de este grupo etario a partir de 2010.

Esta extensión del promedio de vida requiere ajustes en todos los aspectos de la sociedad, principalmente en lo que se refiere al ámbito educacional, una vez que impacta en todos los procesos sociales. Lo que ocurre es que la sociedad discrimina a los viejos, puesto que existe la creencia de que esta etapa es de deterioro y que viejo no aprende más. Pero, a pesar de todos los prejuicios y mitos con relación al envejecimiento, las personas que envejecen están procurando nuevos modos de vida e intentan redefinir sus papeles en la sociedad, lo que redunda en un nuevo concepto de vejez y envejecimiento.

Mariuza Pellozo Lima (2001), maestra en Gerontología de la PUC/São Paulo, comenta sobre la contribución de la neurociencia acerca de la plasticidad del cerebro humano como uno de los avances responsables por el nuevo paradigma. Según ella, "inaugura-se uma nova era para a ciência denominada pela ONU como a década do cérebro”. La nueva descubierta lanzará bases para el estudio de su funcionamiento y potencial, de ahí se vislumbra la posibilidad de que el órgano se regenera continuadamente incluso en la vejez (LIMA, 2001, p. 28)

A causa de esta nueva visión, cabe destacar la importancia de la educación a lo largo de la vida como una de las posibilidades de inclusión y de recuperación del locus social de las generaciones mayores en la sociedad. Muestra de ello son las universidades de Brasil y de otros países que vienen en parte atendiendo a esa demanda a través de programas y proyectos los más diversos, y siguen, en la mayoría de las veces, los objetivos del profesor Pierre Vellas de la Universidad de Toulouse, en Francia: sacar a los mayores del aislamiento, mejorar la salud, energía, interés por la vida y modificar su imagen delante la sociedad.

En Brasil, de acuerdo con Cachioni y Neri (2004) el trabajo educacional para la tercera edad en la universidad fue iniciado en 1982 por la Universidade Federal de Santa Catarina (UFSC) que fundó el Núcleo de Estudos da Terceira Idade (NETI), a partir de la concepción y principio de que el hombre tiene la posibilidad de aprender a lo largo de toda la vida. Además de eso, se basa en el entendimiento de que el que aprende 
debe ser el protagonista de su proceso de vivir, y cuando despertado para la acción gerontológica es un colaborador por excelencia.

De modo general, los programas y proyectos llevados a cabo por el Núcleo de Estudios de la Tercera Edad/UFSC están organizados en forma de módulos. En lo que respeta a los Cursos de Español ofertados a este público específico, su organización es semestral y los niveles son de I a VI, en el periodo de tres años, generalmente durante los periodos lectivos y en el mismo espacio físico en que se dan las clases de los demás cursos regulares de la Universidad, en este caso en el Centro de Comunicación y Expresión, del que forma parte El Curso de Letras Extranjeras/Español. Para que los alumnos se matriculen en los cursos de español la única exigencia hecha es la de que tengan más de cincuenta años de edad.

Los alumnos, después de la conclusión de los seis niveles del Curso, son diplomados o reciben documentos de participación, sin embargo, ninguno se certifica profesionalmente, por tratarse de un Curso de Lenguas que tiene como uno de sus objetivos comunicarse en el idioma extranjero, en este caso la lengua española.

\section{Presupuestos y Principios}

Conforme Both (2002, p.1107) para adelantar en las posibilidades para los mayores se hace necesario buscar respuesta para indagaciones cómo:

- ¿Qué expectativas educacionales caben en esta etapa de vida?

- ¿Cómo aprenden los mayores?

- ¿El aprendizaje tiene el mismo significado en cualquier edad?

- ¿Sobre qué presupuestos teóricos se fundamenta el aprendizaje? (Both, 2002, p.1107)

El autor apunta también, tres principios que deben sostener una propuesta pedagógica para los adultos mayores:

- Principio de la actividad: la actividad educativa contribuye para que la persona mayor se mantenga activa

- Principio de la independencia: la educación debe preparar al adulto mayor para mantener su independencia y autonomía en el más alto grado en relación a los varios niveles tanto familiares como sociales, evitando que sea un simple receptor.

- Principio de la participación: debe ser respectado a lo largo de la vida su derecho a la interacción y participación en todos los niveles de la sociedad.

Se presupone, sobretodo, que aprender hace parte de la naturaleza humana, pero aquí en este estudio nos referimos a un aprendizaje con carácter especifico, fuera de la estructura educacional tradicional. Lo cierto es que de acuerdo con Dohmen (1977) las personas mayores desean continuar a aprender y ser capaces de ello, pero en esta etapa de la vida no necesitan estar limitados a currículos y planes hechos para los jóvenes, ya que poseen un acervo proprio de conocimientos previos y tienen capacidad para adoptar una postura crítica, siendo su aprendizaje voluntario pues nace de sus intereses y necesidades personales.

De esta forma, Dohmen (1977) sugiere que los programas y proyectos sean concebidos y organizados con una metodología particular y bajo la influencia de las varias inferencias de vida de los alumnos. Y en este sentido, Gómes, (2011) profesora de la Universidad Nacional de la Tercera Edad de Lomas de Zamora, Argentina, refuerza los conceptos de Dohmen (1977) al afirmar que los adultos mayores tienen la misma capacidad de aprender que en otras épocas, pero las variaciones obedecen a conocimientos previos, tiempos de cada uno, interés y motivaciones. En este sentido, Dohmen (1977) refuerza que aprender en edad adulta o vejez depende en gran medida de las condiciones del aprendizaje. 


\section{El proyecto de español para tercera edad NETI/UFSC: Procedimientos y Metodología}

Aprender en Legua Extranjera (LE/L2) en la vejez se presenta para el alumno como una gran oportunidad de estar en la Universidad, además de la experiencia de volver a estudiar y de que es capaz de aprender, lo que mejora en mucho su auto estima.

Inicialmente, los objetivos y la metodología del proyecto iniciado en el año de 2004 fueron planeados con el fin de medir la capacidad de aprendizaje de una lengua extranjera después de la adolescencia, una vez que algunas teorías lingüísticas ponen en duda la capacidad de aprender una L2 después de esta etapa de la vida.

La metodología diseñada para el curso inicial se basaba en una perspectiva interaccionista del método comunicativo, semejante a la metodología usada por la escuela tradicional, y sugería las actividades didácticas de un libro del mercado editorial.

El primer grupo de alumnos con que trabajamos era compuesto de doce mujeres y apenas un hombre, con un promedio de edad de 63 años. La gran mayoría estaban jubilados y tenían una variada gama de profesiones y puestos de trabajo, desde amas de casa hasta personas con post grado. Todos, desde las primeras clases, se mostraron muy interesados, pero sus aspiraciones y curiosidades no eran satisfechas a razón de los contenidos presentes en los libros utilizados en clase.

Con esta primera experiencia y frente a un material poco interesante, que no atendía a las necesidades del público que teníamos, buscamos repensar el abordaje y las actividades para alcanzar las metas didácti$\mathrm{co} /$ pedagógicas establecidas como también satisfacer a los alumnos y sus aspiraciones. Fue entonces que, a partir de un segundo momento, con la ayuda del Núcleo de Suporte Pedagógico para Professores de Línguas Estrangeiras (NUSPPLE/ UFSC) empezamos a rediseñar el Curso. Primeramente, a través de un perfil del público albo, hicimos un diagnóstico para determinar los temas de mayor interés por parte de los alumnos, como también las estrategias de aprendizaje y estilos cognitivos de los sujetos, etc., pasamos a adoptar la metodología utilizada en los proyectos desarrollados en el NUSPPLE, que se pauta en los géneros textuales y los mapas semánticos como eje central y que nortean las actividades.

Así, las actividades pasaron a ser elaboradas a partir del eje temático - Salud Física y Mental - que resultó del sondaje hecho con los alumnos, subdividido en los temas de mayor interés respetando los intereses de ellos. Los contenidos, actividades y estrategias estructurados en torno al Mapa Semántico llevan en cuenta la valorización del conocimiento previo y la edad del grupo.

Con ese principio, las prácticas didáctico pedagógicas empleadas en el curso requieren materiales construidos a través de recortes de libros, periódicos, internet, películas, músicas, y otras fuentes variadas. Desde aquel entonces hasta el presente momento, 2004/2012, esta práctica metodológica se construye, se renueva y se perfecciona en cada semestre.

Con el éxito del proyecto, que une investigación, extensión y enseñanza, la enseñanza de español para la tercera edad también pasó a ser desarrollado a partir 2010 por alumnos de la Carrera de Letras Español en la modalidad a distancia (EaD/UFSC) en los polos de apoyo presencial de Foz do Iguaçu/PR y Pato Branco/PR. Los académicos utilizan también la misma metodología y las actividades, como también siguen las mismas propuestas didácticas materiales, y son orientados por las profesoras del NUSPPLE y los miembros que integran el Proyecto, haciendo las adaptaciones pertinentes y necesarias a los grupos y entorno socio cultural.

A partir de la experiencia relatada, llevándose en cuenta toda la duración del proyecto (2004/20012) y con el fin de registrar todo el trabajo de pesquisa, preparación y aplicación del material a lo largo de los 8 años estamos preparando un manual didáctico que será publicado en el primer semestre de 2013, puesto que no hay en el mercado editorial brasileño material específico para el público con el que trabajamos.

La elaboración de dicho manual es un trabajo conjunto entre NUSPPLE y NETI, y es llevado a cabo por dos de las profesoras que ministran las clases de español para la tercera edad. Todo el material que sirve de apoyo y consulta para las clases y elaboración del libro fue recolectado, siendo adaptado de diferentes 
fuentes, desde el inicio del proyecto para después de ser aplicados en las clases, por fin, ser analizados y evaluados en términos de eficiencia y adecuación a los objetivos y contextos de vida en que se desenvuelven. Vale resaltar que en este manual y en la metodología adoptada la gramática no es el foco, puesto que el entendimiento de las Coordinadoras del Proyecto y del Núcleo es el de que la gramática debe ser una herramienta, un elemento integrador para el uso de la lengua, para que se logre la comunicación en el idioma extranjero y no al revés. (COSTA E VIEIRA, 2012)

El curso, actualmente, tiene la duración de tres años divididos en semestres y debido a la metodología adoptada, con material hecho para un fin específico, despierta gran interés por parte de la comunidad y muchas veces no se consigue atender a toda la demanda existente.

\section{Consideraciones finales}

La educación es un derecho fundamental no sólo para la infancia y la juventud, sino que abarca todos los ámbitos de la vida humana. El concepto de educación a lo largo de la vida puede ser concebido como condición para el desarrollo en armonía de la persona y sobretodo hoy debe ser considerado un bien necesario para que los mayores puedan tener una vida plena y sana.

La jubilación, en la actualidad, representa un largo periodo en la vida y es importante que el adulto mayor pueda seguir educándose, bien sea por el simple placer de aprender y de mantener la mente en permanente actividad o como medio de socialización.

El concepto de la educación a lo largo de toda la vida fue formulado por primera vez por los miembros de la Comisión Internacional sobre la Educación para el siglo XXI en su informe titulado «La educación encierra un tesoro", comúnmente llamado Informe Delors.

Con este principio, el aprendizaje de idiomas es una herramienta multiusos ya que, en un mundo cada vez más globalizado, favorece la interculturalidad y el respeto social, ayuda a abrir los horizontes y a relacionarse socialmente. En lo que respeta a la enseñanza aprendizaje de lenguas para los adultos mayores puede desempeñar un papel importante en las sociedades, principalmente en lo que se refiere a dejar claro que estas personas pueden desempeñar una multitud de tareas socialmente productivas, además de darles más y mayor visibilidade.

Para nosotras, la tarea de enseñar es siempre desafiadora, y ejercer la docencia para el público de este proyecto nos trae, además de un permanente aprendizaje y crecimiento personal, la certeza de que los mayores conservan intactas sus competencias intelectuales, sobretodo si las ejercitan. Queda patente, también, que enseñar a personas con tanta experiencia de vida nos proporciona prácticas y reflexiones fundamentales para nuestra tarea de educar, pues nos hace pensar en todo lo que tenemos que llevar en cuenta a la hora de planificar nuestras clases de ELE.

\section{Referencias}

BOTH, Agostinho. Educação permanente para o trabalho educacional com o adulto maduro e com o idoso. In: Tratado de gerontologia e geriatria. Guanabara Ed., 2002. cap. 136.

CACHIONI, Meire. NERI, Anita Liberalisso. Educação e velhice bem sucedida no contexto das Universidades da Terceira Idade. In: NERI, A.L.; YUADSSUNA, M.S. (Org). Velhice bem sucedida. São Paulo, SP: Papirus Ed., 2004.

Centro Latinoamericano y Caribeño de Demografía. División de Población de la CEPAL. Revisión de 2011. Boletín nº 12, octubre 2011. Naciones Unidas, Santigo de Chile. 
. Quem educa os idosos? Editora Alinea, Campinas, São Paulo, 2003.

COSTA, M.J.R.D.; CAMORLINGA, R.; VIEIRA, V.R.A. Língua Espanhola IV. Florianópolis, SC: EdUFSC, 2012.

DOHMEN, George. ¿Cómo aprenden los adultos? In: Periódico Educación - Instituto de Colaboración Científica. Federal Republica of Germany, 1997.

GÓMES, Alejandra Paula. ¿Cómo Construyen el conocimiento los adultos mayores? Disponible en: http//www.fimte.fac.org.ar/doc/01/gomesa.htm Consulta en: 21/8/2012

\section{Anexos}

\section{Mapa temático das unidades didáticas}

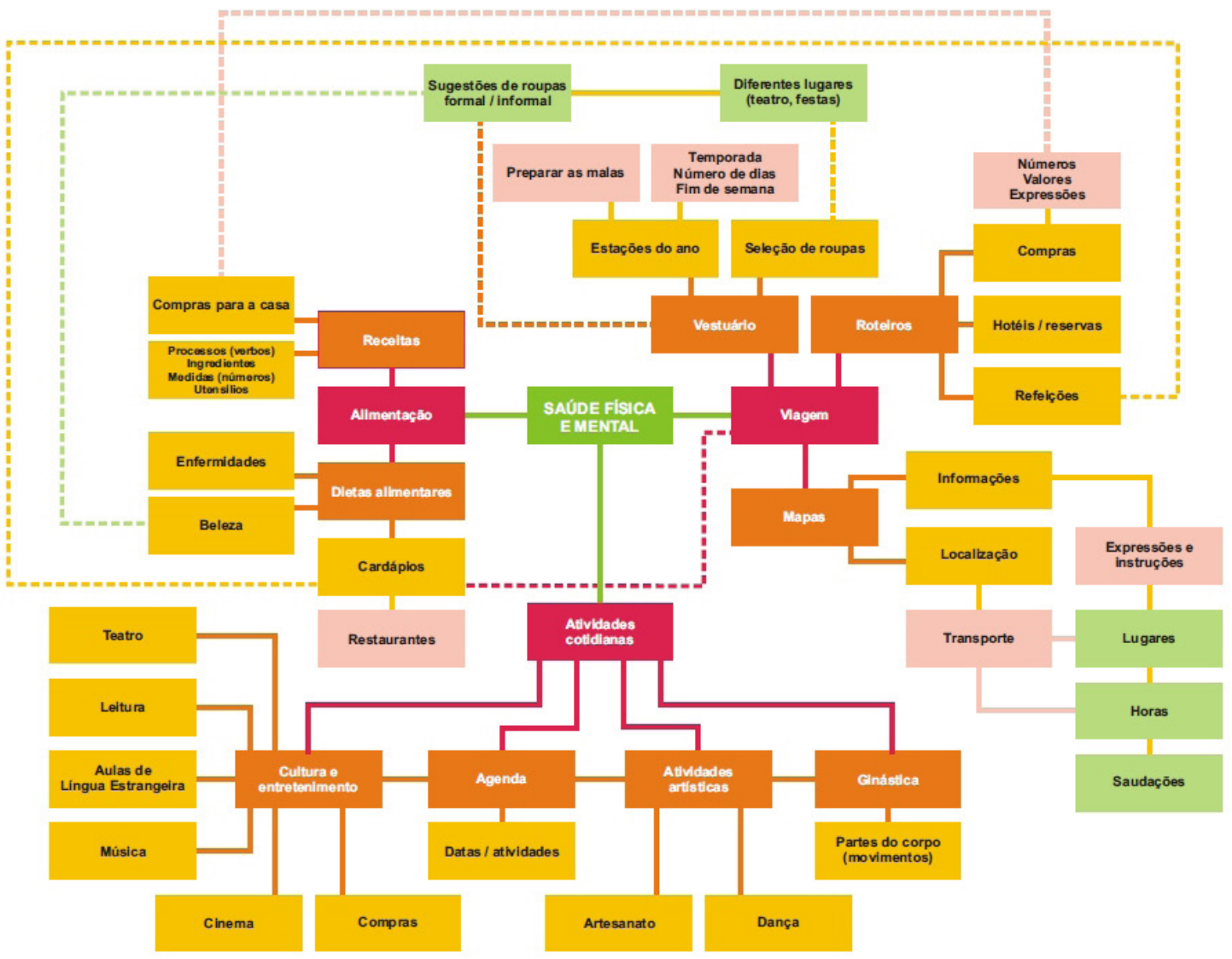

